\title{
SOME EXAMPLES OF INDEFINITE COMPLETE COMPLEX EINSTEIN HYPERSURFACES NOT LOCALLY SYMMETRIC
}

\author{
ALFONSO ROMERO
}

ABSTRACT. Some examples of indefinite complete complex Einstein hypersurfaces of an indefinite complex flat space which are not locally symmetric are given.

Introduction. Recently [3], a systematic study of complex Einstein hypersurfaces in indefinite complex space forms (see [1]) has been made as an extension of the known work of Smyth [5] to indefinite Kaehler metrics. In that paper, the authors found that, except in one case, indefinite complex Einstein hypersurfaces are locally symmetric.

The purpose of this paper is to give some examples of indefinite complex Einstein hypersurfaces of the indefinite complex flat space $\mathbf{C}_{n}^{2 n+1}, n>1$ (see [1]), which are not locally symmetric. In fact, let $M_{p}, p \in \mathbf{Z}, p \geqslant 2$, be the complex hypersurface of $\mathbf{C}_{n}^{2 n+1}$ given by

$$
\sum_{j=1}^{n}\left(z_{j}-z_{n+j}\right)^{p}=p z_{2 n+1} .
$$

Thus, we have a family of spaces $M_{p}$ with several interesting features that we prove in the following

THEOREM. (a) Every $M_{p}$ is a complete complex hypersurface of $\mathbf{C}_{n}^{2 n+1}$ with index $2 n$, which is Ricci-flat but nonflat.

(b) $M_{p}$, with $p>2$, is not locally symmetric and $M_{2}$ is.

(c) If $p \neq p^{\prime}$ then $M_{p}$ and $M_{p^{\prime}}$ are not isometric, but each $M_{p}$ is holomorphically diffeomorphic to $\mathbf{C}^{2 n}$.

(d) If $p>2$, then $M_{p}$ is not homogeneous with respect to the induced Kaehler metric.

We shall use here the same notation as in [3].

1. Preliminaries. For each $z=\left(z_{1}, \ldots, z_{2 n+1}\right) \in M_{p}$

$$
\xi_{z}=\left(\left(\bar{z}_{1}-\bar{z}_{n+1}\right)^{p-1}, \ldots,\left(\bar{z}_{n}-\bar{z}_{2 n}\right)^{p-1},\left(\bar{z}_{1}-\bar{z}_{n+1}\right)^{p-1}, \ldots,\left(\bar{z}_{n}-\bar{z}_{2 n}\right)^{p-1}, 1\right)
$$

Received by the editors November 30, 1984.

1980 Mathematics Subject Classification (1985 Revision). Primary 53C40, 53C50, 53C55.

Key words and phrases. Indefinite complex Einstein hypersurface, locally symmetric, indefinite complex space form. 
is a normal vector to $M_{p}$ in $z$ and satisfies $g\left(\xi_{z}, \xi_{z}\right)=1$, where $g$ is the usual Kaehler flat metric of index $2 n$ on $\mathrm{C}_{n}^{2 n+1}$ (see [1]). Thus, $\xi$ is a unit normal vector field to $M_{p}$.

It is easy to see that the Weingarten endomorphism $A$ associated to $\xi$ is given, in each $z \in M_{p}$, by

$$
\begin{aligned}
& A\left(a_{1}, \ldots, a_{n}, a_{n+1}, \ldots, a_{2 n}, a_{2 n+1}\right) \\
& =\left(f_{1}(z)\left(\bar{a}_{1}-\bar{a}_{n+1}\right), \ldots, f_{n}(z)\left(\bar{a}_{n}-\bar{a}_{2 n}\right),\right. \\
& \left.\quad f_{1}(z)\left(\bar{a}_{1}-\bar{a}_{n+1}\right), \ldots, f_{n}(z)\left(\bar{a}_{n}-\bar{a}_{2 n}\right), 0\right)
\end{aligned}
$$

where $f_{j}(z)=(1-p)\left(\bar{z}_{j}-\bar{z}_{n+j}\right)^{p-2}, j=1,2, \ldots, n$.

Let $P_{j}$ be the tensor field of type $(1,1)$ defined by

$$
\begin{aligned}
& P_{j}\left(a_{1}, \ldots, a_{n}, a_{n+1}, \ldots, a_{2 n}, a_{2 n+1}\right) \\
& \quad=\left(0, \ldots, 0, \bar{a}_{j}-\bar{a}_{n+j}, 0, \ldots, 0, \bar{a}_{j}-\bar{a}_{n+j}, 0, \ldots, 0\right)
\end{aligned}
$$

for $j=1,2, \ldots, n$, where $\left(a_{i}\right)$ denotes any tangent vector to $M_{p}$ in $z$. Then, we can write

$$
A=\sum_{j=1}^{n} f_{j}(z) P_{j}
$$

Moreover, the following properties hold:

$$
\begin{aligned}
& P_{j} \text { is a selfadjoint operator of the tangent space to } M_{p} \text { in } z \text {. } \\
& P_{i} \cdot P_{j}=0, \quad \nabla P_{j}=0 \text { and } J \cdot P_{j}=-P_{j} \cdot J
\end{aligned}
$$

where $\nabla$ and $J$ are, respectively, the metric connection and the complex structure of $M_{p}$.

By using the Gauss equation [3, (1.1)] for the curvature tensor $R$ of $M_{p}$, we obtain

$$
R=\sum_{i, j=1}^{n} f_{i j}(z) R_{i j}^{0}+\sum_{i, j=1}^{n} h_{i j}(z) J R_{i j}^{0}
$$

where $f_{i j}(z)$ and $h_{i j}(z)$ are, respectively, real and imaginary parts of $\overline{f_{i}(z)} f_{j}(z), \overline{f_{i}(z)}$ is the complex conjugate of $f_{i}(z)$ and $R_{i j}^{0}$ the tensor field given by

$$
\begin{aligned}
R_{i j}^{0}(X, Y) Z= & g\left(P_{i} Y, Z\right) P_{j} X-g\left(P_{i} X, Z\right) P_{j} Y \\
& +g\left(J P_{i} Y, Z\right) J P_{j} X-g\left(J P_{i} X, Z\right) J P_{j} Y
\end{aligned}
$$

for all $X, Y, Z$ tangent to $M_{p}$. So,

Taking into account that $\nabla P_{j}=0$, it follows $\nabla R_{i j}^{0}=0$ for all $i, j=1,2, \ldots, n$.

$$
\nabla_{V}^{k} R=\sum_{i, j=1}^{n} V\left(\stackrel{(k)}{\cdots} V\left(f_{i j}\right)\right) R_{i j}^{0}+\sum_{i, j=1}^{n} V\left(\stackrel{(k)}{\cdots} V\left(h_{i j}\right)\right) J R_{i j}^{0}
$$

for all $V$ tangent to $M_{p}, k \in \mathbf{Z}, k \geqslant 1$, where $\nabla_{V}^{k}=\nabla_{V} \stackrel{(k)}{\cdots} \nabla_{V}$. 
2. Proof of the Theorem. From (1.1) we have $A^{2}=0$ but $M_{p}$ is not totally geodesic, and hence, the formula for the Ricci tensor (1.4) in [3] implies that $M_{p}$ is Ricci flat but nonflat. Now suppose that $\gamma=\gamma(t)$ is a geodesic in $M_{p}$. Then components of $\gamma$ (as a curve in $\mathrm{C}_{n}^{2 n+1}$ ) satisfy $\gamma_{j}^{\prime \prime}(t)=\gamma_{n+j}^{\prime \prime}(t)=F_{j}(t), j=$ $1,2, \ldots, n, \gamma_{2 n+1}^{\prime \prime}(t)=G(t)$, where $F_{j}(t), G(t)$ are certain polynomic functions of complex coefficients with degree $\left(F_{j}(t)\right)=2 p-3$ and degree $(G(t))=p-2$. Thus, components of $\gamma(t)$ are also polynomic and hence $\gamma(t)$ is defined for all $t \in \mathbf{R}$. This proves that $M_{p}$ is complete for all $p$, and so we have (a).

For (b) note that functions $f_{i j}$ and $h_{i j}$ in (1.5) are polynomic of degree $2(p-2)$ (in particular constants for $p=2$ ). By using (1.7), we have $\nabla^{2(p-2)+1} R=0$. So, $\nabla R=0$ for $p=2$, that is, $M_{2}$ is locally symmetric. Now, let us suppose $p>2$ and take for fixed $j, X \in \operatorname{Ker} P_{i}, i \neq j, X \notin \operatorname{Ker} P_{j}$. Then, using (1.4), (1.7) becomes

$$
\begin{aligned}
& g\left(\left(\nabla_{V}^{2(p-2)} R\right)(X, J X) J X, X\right) \\
& =-2\left(V\left(\stackrel{2(p-2)}{\cdots} V\left(f_{j j}\right)\right)\right)\left\{g\left(P_{j} X, X\right)^{2}+g\left(J P_{j} X, X\right)^{2}\right\} .
\end{aligned}
$$

If $\nabla^{2(p-2)} R=0$, from (2.1) we obtain $g\left(P_{j} X, X\right)=g\left(J P_{j} X, X\right)=0$ because

$$
V\left(\stackrel{2(p-2)}{\cdots} V\left(f_{j j}\right)\right) \neq 0
$$

But, from (1.2) this implies that $X \in \operatorname{Ker} P_{j}$ and it contradicts our assumption.

From the above discussion, we have $\nabla^{2(p-2)+1} R=0$ and $\nabla^{2(p-2)} R \neq 0$ for all $p$. Then $M_{p}$ and $M_{p^{\prime}}$ cannot be isometric if $p \neq p^{\prime}$. However, $M_{p}$ is the graph of a holomorphic function of $\mathbf{C}^{2 n}$ on $\mathbf{C}$. And this concludes (c).

Finally, we will prove (d). In fact, let us take $z \in M_{p}, p>2$, verifying $z_{j}=z_{n+j}$ for all $j=1,2, \ldots, n$ and $z_{2 n+1}=0$. From (1.5) we have $R=0$ in this point $z$. $M_{p}$ homogeneous implies $R=0$ in every point, but it is not possible because $M_{p}$ is not a flat space.

RemarKs. (1) We know that $M_{p}$ has index $2 n$ and real dimension $4 n$. The change of the Kaehler metric $g$ of the ambient space by its negative permits us to obtain that $M_{p}$ can be also considered as a complex hypersurface of $\mathbf{C}_{n+1}^{2 n+1}$ and so, we can give a similar result to our Theorem for the family $M_{p}$ in $\mathbf{C}_{n+1}^{2 n+1}$.

(2) $M_{2}$ is identical to the space of Example 3.4 in [3] which has a Weingarten endomorphism with maximal rank in each point (see also [2, p. 346]).

(3) Given positive integers $s, m, s \leqslant m$, we have that $M_{p} \times \mathbf{C}_{s}^{m}$ is a complex hypersurface of $\mathbf{C}_{n+s}^{2 n+m+1}$ (or $\mathbf{C}_{n+s+1}^{2 n+m+1}$ ) for all $p \in \mathbf{Z}, p \geqslant 2$. Thus, we can obtain a result analogous to our Theorem for this family of spaces.

(4) In the definite case, Smyth proves in [6] that a complex hypersurface of a complex space form is homogeneous if and only if it is an Einstein space. This is not true here because our Theorem provides us many indefinite complete complex Einstein hypersurfaces that are not homogeneous.

(5) Since every $M_{p}$ satisfies $A^{2}=0$, it is easy to see that the curvature tensor $R$ of $M_{p}$ verifies Nomizu's condition $R \cdot R=0$ in [4]. However, we know by (b) that if $p>2, M_{p}$ is not locally symmetric. So, $R \cdot R=0$ does not imply $\nabla R=0$ for complete complex hypersurfaces of an indefinite complex flat space. 


\section{REFERENCES}

1. M. Barros and A. Romero, Indefinite Kählerian manifolds, Math. Ann. 261 (1982), 55-62.

2. M. Cahen and M. Parker, Sur des classes d'espaces pseudo-riemanniens symmétriques, Bull. Soc. Math. Belg. Sér. A 12 (1970), 339-354.

3. S. Montiel and A. Romero, Complex Einstein hypersurfaces of indefinite complex space forms, Math. Proc. Cambridge Philos. 94 (1983), 495-508.

4. K. Nomizu, On hypersurfaces satisfying a certain condition on the curvature tensor, Tôhoku Math. J. 20 (1968), 46-59.

5. B. Smyth, Differential geometry of complex hypersurfaces, Ann. of Math. (2) 85 (1967), 246-266.

6. Homogeneous complex hypersurfaces, J. Math. Soc. Japan 20 (1968), 643-647.

Departamento de Geometria y Topología, Facultad de Ciencias, Universidad de Granada, 18071-Granada, Spain 